\title{
Social predictors of adult asthma: a co-twin case-control study
}

\author{
E Huovinen, J Kaprio, L A Laitinen, M Koskenvuo
}

\begin{abstract}
Background-Environmental factors are needed to explain the observed increase in the prevalence of asthma during recent decades, despite the existence of a recognised genetic component in asthma. A co-twin case-control study was undertaken to examine possible social risk factors for asthma.

Methods-Asthma diagnoses were based on register data of reimbursed asthma medication. During 17 years follow up of the Finnish twin cohort, 262 twin pairs discordant for incident asthma were identified. Conditional logistic regression for 1-1 matched data was used for risk calculation.

Results-The atopic twin had an increased risk of asthma compared with the non-atopic co-twin (RR 2.91, 95\% CI 1.81 to 4.68$)$. The more educated twin had a decreased risk of asthma compared with his/her twin sibling with less education (RR $0.45,95 \%$ CI 0.23 to 0.86 ), and the twin who participated in conditioning exercise had a decreased risk of asthma compared with the more sedentary cotwin (RR $0.55,95 \%$ CI 0.34 to 0.88 ).

Conclusions-In addition to allergic diseases, educational level and physical activity are associated with adult onset asthma, which indicates a role for factors associated with life style.

(Thorax 2001;56:234-236)
\end{abstract}

Keywords: asthma; twins; social factor; life style

Asthma has a strong genetic component according to twin studies ${ }^{1}$ and molecular genetic studies are providing insight into relevant genes. ${ }^{2}$ However, this genetic susceptibility for asthma is unlikely to explain the observed increase in the prevalence of asthma during recent decades, and environmental explanations are needed. In a twin study it is possible to study environmental factors while taking into account the genetic component of asthma. Twin pairs are matched either fully (monozygotic, MZ) or partly (dizygotic, DZ) on genetic make up, and generally also share childhood exposures. We used a co-twin case-control design to examine possible social and lifestyle determinants of adult asthma.

\section{Methods}

STUDY POPULATION

The Finnish twin cohort is a population based sample of like-sex adult twin pairs selected from the Central Population Registry of Finland as pairs of individuals with the same birth date and sex, the same surname, and local community at birth. ${ }^{3}$ A health questionnaire was mailed to the cohort members in the autumn of 1975 with a question whether asthma had ever been diagnosed by a physician. The response rate was $89 \%$.

In Finland, National Sickness Insurance (NSI) has covered $80-100 \%$ of the costs of drug treatment for chronic bronchial asthma since 1970 (including corticosteroids, corticotrophins, cromoglycate, sympathomimetics, theophylline and its derivatives, hydroxyzine, ipratropium, oxytropium, nedocromil, and some combinations). A prerequisite for being registered is an accurate diagnosis of asthma by a specialist in allergy, pulmonary diseases, internal medicine, or paediatrics, or a diagnosis based on an appropriate hospital examination and acceptance by a consultant physician from the Social Insurance Institution of Finland. ${ }^{4}$ The National Board of Health maintains a register of all patients discharged from the hospital in Finland since 1969, including their diagnoses. ${ }^{5}$ Permissions for the use of register data were obtained from the national authorities. Data from the registers of the Social Insurance Institution and National Board of Health were obtained by linking the unique social security numbers given to all residents in Finland.

Respondents reporting asthma or with missing data on asthma or on smoking in the 1975 questionnaire, patients entitled to reimbursement for asthma medication and those treated in hospital for asthma before 1977 were excluded $(n=1546)$. This population, initially free of asthma, was then confined to twins alive at the beginning of 1977 aged 20-50 in 1977 $(n=20472)$. Twin pairs discordant for the right to reimbursed asthma medication in 197793 - that is, the other twin had that right while his/her twin sibling did not-were then identified (318 pairs) and three pairs were further excluded because the non-medicated twin had been treated in hospital for asthma during 


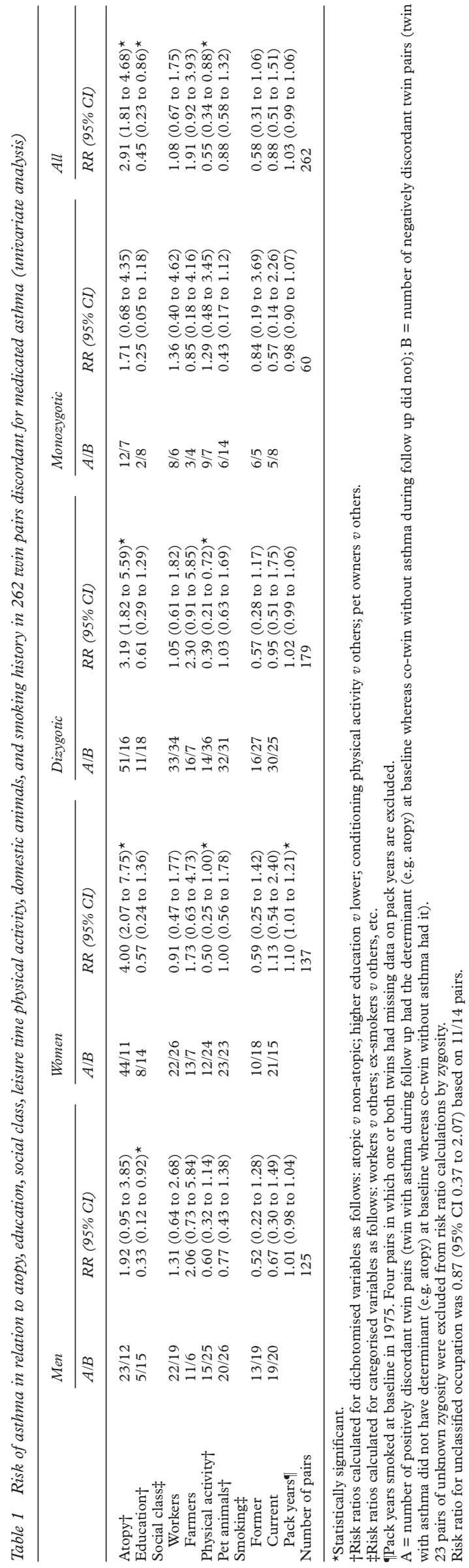

follow up. In addition, 18 twin pairs were concordant for asthma medication.

The studied variables were chosen from available data of the Finnish twin cohort on the grounds of previous findings. A history of atopic diseases was based on whether allergic rhinitis (including hay fever), allergic eczema, or urticaria had ever been diagnosed by a physician. Respondents were classified as nonsmokers (including occasional smoking), former or current smokers. The number of cigarettes smoked daily was asked separately for former and current smokers. ${ }^{3}$ Social class was based on occupation: upper and lower professionals, skilled and unskilled workers, farmers and unclassified (students, conscripts, housewives, etc). ${ }^{3}$ Education was measured by the schooling completed by the respondent (nine alternatives) and divided into lower (at most, primary school for 6-7 years and possibly some vocational training) and higher (at least, junior high school for 9 years) grades. Physical activity was measured by a series of questions about leisure time physical activities. ${ }^{6}$ They were also asked if there were any domestic animals in the household. Standard questionnaire assessment of chronic bronchitis and dyspnoea was used. ${ }^{3}$

DATA ANALYSIS AND STATISTICAL METHODS

Within the cohort nested case-control analyses were conducted among twin pairs discordant for incident asthma and with no missing data on study variables ( $n=262$ pairs) to examine whether the risk of asthma in cases in relation to chosen determinants differed from the risk of their twin siblings without that determinant. The significance of the relative risk was assessed by McNemars' two sided test (dichotomous variables) and by Bowkers' test of symmetry (multicategorical variables). Conditional logistic regression for 1-1 matched data was then used to calculate risk ratios and their confidence limits to find out the difference in the asthma risk of the twin with the determinant in question (for example, atopy) compared with that of his/her co-twin without that determinant. ${ }^{7}$ All statistical analyses were computed by SAS (SAS Institute, Cary, NC, USA).

\section{Results}

There were significant differences between asthmatic and non-asthmatic co-twins in atopy, education, and physical activity but not in occupation or pet possession. Significant differences in the numbers of cigarettes smoked were observed between asthma discordant co-twins only among women $(\mathrm{p}=0.02$; table 1). Adjusting for atopy or for other social variables did not change risk ratios. Risks calculated among twin pairs initially also free of chronic bronchitis and dyspnoea were similar in magnitude, although no longer statistically significant for education (RR $0.50,95 \%$ CI 0.20 to 1.24 ) and physical activity (RR 0.60 , $95 \%$ CI 0.33 to 1.08 ).

\section{Discussion}

In this study we have used as the outcome asthma needing drug treatment as diagnosed 
by specialists. The strength of this kind of definition is the consistent criteria on which the diagnoses are based and the independence from questionnaire responses but, on the other hand, it excludes milder cases. Adult patients diagnosed as having asthma can in some cases have chronic obstructive pulmonary disease (COPD). According to the criteria of the Social Insurance Institution, these patients also had to benefit from asthma medication and thus they probably had an asthmatic component in their disease. Other strengths of the study are its prospective nature and the use of twins. Comparison within twin pairs adjusts for most childhood factors such as diet, allergen exposure, passive smoking, and parental characteristics in addition to genetic factors.

Educational level probably describes the way of life, while occupation defines work exposures better. In this study education had a protective effect against asthma while occupation had almost no effect. Top ranking athletes have been shown to have higher asthma prevalences than the general population, ${ }^{8}$ while former elite athletes show no increase in lifetime occurrence of asthma. ${ }^{9}$ We found a lower risk of asthma in twins undergoing conditioning exercise compared with the sedentary twin.

The association between smoking and asthma is complex..$^{10}$ Especially among the adult population, the diagnosis of asthma may also include subjects with smoking related airway obstructions. We found an association between the risk of asthma and the amount of cigarettes smoked only in women.
In addition to atopy, educational level and physical activity appear to be associated with onset of asthma in adults independent of childhood exposures. Thus, studies on living conditions and on factors altering host resistance are needed to clarify these findings and the mechanisms on which they are based.

The work was performed at the Department of Public Health, University of Helsinki. This study was supported by the Ida Montin Foundation, Academy of Finland (grants 38332, 42044).

1 Koppelman GH, Los H, Postma DS. Genetic and environment in asthma: the answer of twin studies. Eur Respir $\mathcal{F}$ 1999;13:2-4.

2 Holgate ST, Bleecker ER, Meyers DA, et al. The genetics of asthma:methodological approaches. Clin Exp Allergy 1998; 28:Suppl 1

3 Huovinen E, Kaprio J, Vesterinen E, et al. Mortality of adults with asthma: a prospective cohort study. Thorax 1997;52:49-54.

4 Vesterinen E, Pukkala E, Timonen T, et al. Cancer incidence among 78,000 asthmatic patients. Int $\mathcal{F}$ Epidemiol 1993;22: 976-82.

5 Keskimäki I, Aro S. Accuracy of data on diagnosis, procedures and accidents in the Finnish hospital discharge register. Int 7 Health Sci 1991;2:15-21.

6 Kujala UM, Kaprio J, Sarna S, et al. Relationship of leisuretime physical activity and mortality, the Finnish twin time physical activity and mort

cohort. $\mathcal{F} A M A$ 1998;279:440-4.
7 Duffy DL. The co-twin control study. In: Spector TD, Snieder H, MacGregor AJ, eds. Advances in twin and Snieder H, MacGregor AJ, eds. Advances in twin and
sib-pair analysis. London: Oxford University Press, 2000: 53-66

8 Weiler JM, Layton T, Hunt M. Asthma in United States Olympic athletes who participated in the 1996 summer games. F Allergy Clin Immunol 1998;102:722-6.

9 Kujala UM, Sarna S, Kaprio J, et al. Asthma and other pulmonary diseases in former elite athletes. Thorax 1996;51: $288-92$.

10 Strachan DB, Butland BK, Anderson HR. Incidence and prognosis of asthma and wheezing illness from early childhood to age 33 in a national British cohort. BMF 1996;312: 1195-9. 